\title{
On enhancing SIMD-controlled DSPs for performing recursive filtering
}

\author{
Michael Hosemann · Gerhard P. Fettweis
}

Published online: 13 July 2006

(C) Springer Science + Business Media, LLC 2006

Abstract In the print and online version of the original article, the authors' biographies and photographs were mistakenly omitted. Their biographies and photos are included below.

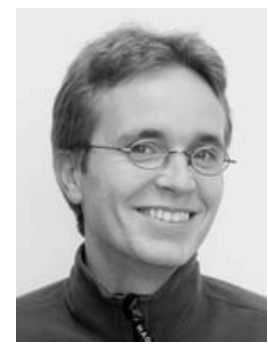

Michael Hosemann studied electrical engineering at Technische Universitaet Dresden, Germany and graduated in 2000 after one year as a research scholar at the MPRG, Virgina Tech, Blacksburg, U.S.A. with a Diplomingenieur degree. From 2000 to 2004 he was with the Vodafone Chair for Mobile Communications Systems at Technische Universitaet in Dresden, Germany where he undertook research towards a Ph.D. His research interests are in signal processing systems and their implementation in hardware. In 2004 he worked for Signalion GmbH, Dresden and is now a Systems Design Engineer with Frontier Silicon, Cambridge, U.K.

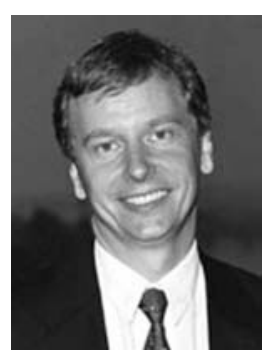

Gerhard P. Fettweis studied electrical engineering at the Aachen University of Technololgy (RWTH) in Germany and earned a PhD degree in 1990. From 1990 to 1991, he was Visiting Scientist at the IBM Almaden Research Center in San José CA, developing signal processing innovations for IBMs disk drive products. From 1991 to 1994, he was a Scientist with TCSI Inc., Berkeley, CA, responsible for signal processor development projects for mobile phone chip-sets. Since September 1994 he holds the Vodafone Chair at the Technische Universitaet in Dresden, Germany. Also, he is the co-founder of four successful start-ups which where spun out of his group: Systemonic AG, now Philips Semiconductors Dresden, Radioplan, Signalion, and In-Circuit.

The online version of the original article can be found at:

http://dx.doi.org/10.1007/s11265-006-7266-2. 